\title{
THE TENSOR PRODUCT OF COMMUTATIVE SEMIGROUPS
}

\author{
BY \\ PIERRE ANTOINE GRILLET
}

In an earlier paper we defined and proved some properties of the tensor product of semigroups. It turns out that the category of commutative semigroups has also a tensor product, which is in many respects more interesting. It keeps all the main properties of the tensor product of arbitrary semigroups (preservation of one-to-one consistent homomorphisms, right exactness, adjoint associativity) and is furthermore colimit-preserving and an associative operation; when applied to abelian groups, it gives the ordinary tensor product of groups.

These properties occupy the second section of this paper, the first being devoted to some examples. In the third section, we study flat commutative semigroups. We prove that a flat commutative semigroup with a minimal generating subset must be free, but that flatness is not hereditary and that there exist flat commutative semigroups which are not free. The nature of flat commutative semigroups remains open, as well as the noncommutative case.

Heavy use is made of the properties of the tensor product of arbitrary semigroups, which we established in [5]. For the fundamentals of semigroup theory, the reader is referred to [1] and [2]. Throughout, the largest commutative homomorphic image of a semigroup $A$ will be denoted by $C(A)$; the largest idempotent (resp. normal) homomorphic image of $A$ will be denoted by $E(A)$ (resp. $N(A)$ ) (normal means: $(x y)^{n}=x^{n} y^{n}$ for all $x, y \in A$ and all $n$ [5], [8]). These are covariant functors, in fact coreflexions, of the category of all semigroups onto the full subcategory under consideration; as coreflexions, they preserve colimits and, for instance, the semigroups Hom $(A, B)$ and $\operatorname{Hom}(C(A), B)$ are naturally isomorphic whenever $B$ is commutative (if $B$ is commutative, $\operatorname{Hom}(A, B)$ is the (commutative) semigroup of all homomorphisms of $A$ into $B$ with pointwise multiplication; for coreflexions, see [6]).

1. Definition, existence, examples. If $\left(A_{i}\right)_{i \in I}$ is a family of semigroups, we say that a mapping $s$ of the cartesian product $\prod_{i \in I} A_{i}$ into some semigroup $C$ is I-linear if for every $i_{0} \in I$ the mapping $a_{i_{0}} \leadsto s\left(\left(a_{i}\right)_{i \in I}\right)$ is a homomorphism of $A_{i_{0}}$ into $C$ whenever all $a_{i}$ are fixed except $a_{i_{0}}$. If all the $A_{i}$ 's are commutative and if an $I$-linear mapping $t$ of $\prod_{i \in I} A_{i}$ into some commutative semigroup $T$ is such that any $I$-linear mapping $s$ of $\prod_{i \in I} A_{i}$ into any commutative semigroup $C$ has the form $u \circ t$ for some unique homomorphism $u$ of $T$ into $C$, then we say that the pair $(t, T)$ (or the

Received by the editors November 20, 1967 and, in revised form, April 1, 1968. 
semigroup $T)$ is a commutative tensor product of the family $\left(A_{i}\right)_{i \in I}$. In such case, we shall denote $T$ and $t\left(\left(a_{i}\right)_{i \in I}\right)$ by $\bigotimes_{i \in I}^{c} A_{i}$ and $\bigotimes_{i \in I}^{c} a_{i}$, or by $A_{1} \otimes^{c} A_{2} \otimes^{c} \cdots \otimes^{c} A_{n}$, $a_{1} \otimes^{c} a_{2} \otimes^{c} \cdots \otimes^{c} a_{n}$ if $I=\{1,2, \ldots, n\}$.

By dropping all commutativity restrictions in the definition above, we obtain the tensor product $\otimes$ in the category of all semigroups. We use the notation $\otimes^{c}$ for the commutative tensor product because $\bigotimes_{i \in I} A_{i}$ need not be a commutative semigroup even when all $A_{i}$ 's are commutative (Example 2.3 of [5]), hence need not coincide with $\bigotimes_{i \in I}^{c} A_{i}$.

The method used in [5, Theorem 2.1] to prove the existence of $\bigotimes_{i \in I} A_{\mathfrak{i}}$ works in any variety and can thus be used to establish the existence of the commutative tensor product. However, the following method gives a very useful relationship between our two tensor products.

THEOREM 1.1. Let $\left(A_{i}\right)_{i \in I}$ be any family of commutative semigroups. There exists a commutative tensor product of the family $\left(A_{i}\right)_{i \in I}$ and it is unique up to isomorphism. Furthermore, $\bigotimes_{i \in I}^{c} A_{i} \cong C\left(\bigotimes_{i \in I} A_{i}\right)$.

Proof. The uniqueness is immediate from the definition by universal property. For the existence, let $T=C\left(\bigotimes_{i \in I} A_{i}\right), p$ be the projection of $\bigotimes_{i \in I} A_{i}$ onto $T$ and $t$ be defined by $t\left(\left(a_{i}\right)_{i \in I}\right)=p\left(\bigotimes_{i \in I} a_{i}\right)$. Then $t$ is $I$-linear. If $s$ is an $I$-linear mapping of $\prod_{i \in I} A_{i}$ into a commutative semigroup $C$, then there is a homomorphism $v$ of $\bigotimes_{i \in I} A_{i}$ into $C$ such that $s\left(\left(a_{i}\right)_{i \in I}\right)=v\left(\bigotimes_{i \in I} a_{i}\right)$ identically; since $C$ is commutative, $v$ factors through $p, v=u \circ p$ and now $s\left(\left(a_{i}\right)_{i \in I}\right)=u\left(t\left(\left(a_{i}\right)_{i \in I}\right)\right)$ identically. Finally $T$ is generated by the elements of the form $t\left(\left(a_{i}\right)_{i \in I}\right)$, since $\bigotimes_{i \in I} A_{i}$ is generated by the elements of the form $\bigotimes_{i \in I} a_{i}$; hence the homomorphism $u$ is unique. This completes the proof.

As a consequence, the two following properties of $\otimes$ can be extended to $\otimes^{c}$ : namely, $E \otimes A \cong E(A)$ if $E$ is a one element semigroup, $N \otimes A \cong N(A)$ if $N$ is an infinite cyclic semigroup (Propositions 1.1, 1.2 of [5]). If $A$ is commutative, then $E \otimes^{c} A \cong C(E \otimes A) \cong C(E(A)) \cong E(A), N \otimes^{c} A \cong C(N \otimes A) \cong C(N(A)) \cong A$. Observe that $E(A)$ is now the largest semilattice homomorphic image of $A$; it is trivial if and only if $A$ is archimedean [1]. As for $N \otimes A \cong A$, the isomorphisms are given by $a \leadsto a \otimes^{c} b, b^{n} \otimes a \rightsquigarrow a^{n}$, if $b$ is the generator of $N$; they can be used for a direct proof.

The following two properties, however, do not hold for $\otimes$. Example 2.3 of [5] shows that $A \otimes B$ need not be finite when $A$ and $B$ are finite. For $\otimes^{c}$, we have

Proposition 1.2. If $A$ is a finite commutative semigroup and if $B$ is a finitely generated commutative semigroup, then $A \otimes^{c} B$ is finite.

Proof. Let $S$ be a finite generating subset of $B$; then $A \otimes^{c} B$ is generated by all elements of the form $a \otimes^{c} s(a \in A, s \in S)$ hence is finitely generated. On the other hand, each element $a$ of $A$ has finite order, say $a^{r}=a^{r+p}(r, p>0)$, whence every generator of $A \otimes^{c} B$ has finite order since, by bilinearity, 


$$
\left(a \otimes^{c} s\right)^{r}=a^{r} \otimes^{c} s=a^{r+p} \otimes^{c} s=\left(a \otimes^{c} s\right)^{r+p} .
$$

Since $A \otimes^{c} B$ is commutative, it must now be finite.

Proposition 1.3. Let $A$ and $B$ be abelian groups. Then $A \otimes^{c} B$ is an abelian group, namely the tensor product of $A$ and $B$ as abelian groups.

Proof. By [5, Proposition 1.4], $A \otimes B$ is a group. Thus $A \otimes^{c} B$ is an abelian group by 1.1. Finally, the universal property which characterizes the tensor product of abelian groups follows for $A \otimes^{c} B$ from its very definition.

We conclude with two more examples which will be useful later.

Proposition 1.4. Let $Z$ be the additive group of all integers and $A$ be any commutative semigroup. Then $Z \otimes^{c} A$ is union of groups.

Proof. We know that $Z \otimes^{c} A$ is generated by all elements of the form $n \otimes^{c} a$. By bilinearity

$$
\begin{aligned}
\left(n \otimes^{c} a\right)\left(m \otimes^{c} b\right) & =\left(1 \otimes^{c} a^{n} b^{m}\right) & & \text { if } n, m>0, \\
& =\left(-1 \otimes^{c} a^{-n} b^{-m}\right) & & \text { if } n, m<0
\end{aligned}
$$

so that the elements of $Z \otimes^{c} A$ can be written under one of the two forms $n \otimes^{c} a$, $\left(n \otimes^{c} a\right)\left(m \otimes^{c} b\right)$. For a given $a$, the elements of the form $n \otimes^{c} a$ form a group, homomorphic image of $Z$. For given $a$ and $b$, the elements of the form $\left(n \otimes^{c} a\right)\left(m \otimes^{c} b\right)$ form also a group; indeed $\left(0 \otimes^{c} a\right)\left(0 \otimes^{c} b\right)=0 \otimes^{c} a b$ is idempotent and

$$
\begin{aligned}
\left(n \otimes^{c} a\right)\left(m \otimes^{c} b\right)\left(0 \otimes^{c} a\right)\left(0 \otimes^{c} b\right) & =\left(n \otimes^{c} a\right)\left(m \otimes^{c} b\right), \\
\left(n \otimes^{c} a\right)\left(m \otimes^{c} b\right)\left(-n \otimes^{c} a\right)\left(-m \otimes^{c} b\right) & =\left(0 \otimes^{c} a\right)\left(0 \otimes^{c} b\right)
\end{aligned}
$$

for all $n, m \in Z$. This completes the proof.

If $A$ and $B$ are any commutative semigroups, $A \otimes B$ can be presented as the semigroup generated by all $a \otimes b$ subject to all bilinearity relations: $a \otimes b b^{\prime}$ $=(a \otimes b)\left(a \otimes b^{\prime}\right), a a^{\prime} \otimes b=(a \otimes b)\left(a^{\prime} \otimes b\right)$ (cf. [5]). By Theorem 1.1, $A \otimes^{c} B$ can therefore be presented as the commutative semigroup generated by all $a \otimes^{c} b$ subject to all bilinearity relations. If $A$ and $B$ are themselves given by generators and relations, the presentation of $A \otimes^{c} B$ is correspondingly simplified. We use this technique to construct the next example.

Proposition 1.5. Let $N_{0}$ be the additive semigroup of all nonnegative integers. Let $A$ be any commutative semigroup. Set $B=E(A), C=A \times B, M=A \cup B \cup C$ and let $p$ be the projection of $A$ onto $B$. Define a multiplication in $M$ by

$$
\begin{aligned}
a^{\prime} * a^{\prime \prime} & =a^{\prime} a^{\prime \prime}, \quad b^{\prime} * b^{\prime \prime}=b^{\prime} b^{\prime \prime}, \quad\left(a^{\prime}, b^{\prime}\right) *\left(a^{\prime \prime}, b^{\prime \prime}\right)=\left(a^{\prime} a^{\prime \prime}, b^{\prime} b^{\prime \prime}\right), \\
a * b & =b * a=(a, b), \quad a *\left(a^{\prime}, b\right)=\left(a^{\prime}, b\right) * a=\left(a a^{\prime}, b\right), \\
b *\left(a, b^{\prime}\right) & =\left(a, b^{\prime}\right) * b=\left(a, b b^{\prime}\right)
\end{aligned}
$$

for all $a, a^{\prime}, a^{\prime \prime} \in A, b, b^{\prime}, b^{\prime \prime} \in B$. Let $\mathscr{C}$ be the smallest congruence on $M$ such that $a \mathscr{C}(a, p(a))$ for all $a \in A$. Let $T=M / \mathscr{C}$ and $k$ be the projection of $M$ onto $T$. Then 
$N_{0} \otimes^{c} A \cong T$ and the isomorphism sends $0 \otimes^{c} a$ onto $k(p(a))$ and $n \otimes^{c} a$ onto $k\left(a^{n}\right)$ $(n>0)$.

Proof. The semigroup $N_{0} \otimes^{c} A$ is the commutative semigroup generated by all elements $0 \otimes^{c} a(=p(a)), 1 \otimes^{c} a(=a)$ subject to the following relations:

$$
\begin{aligned}
& \left(0 \otimes^{c} a^{\prime}\right)\left(0 \otimes^{c} a^{\prime \prime}\right)=0 \otimes^{c} a^{\prime} a^{\prime \prime}, \quad\left(0 \otimes^{c} a\right)^{2}=0 \otimes^{c} a, \\
& \left(1 \otimes^{c} a^{\prime}\right)\left(1 \otimes^{c} a^{\prime \prime}\right)=1 \otimes^{c} a^{\prime} a^{\prime \prime} \quad \text { and } \quad\left(0 \otimes^{c} a\right)\left(1 \otimes^{c} a\right)=1 \otimes^{c} a .
\end{aligned}
$$

Clearly $M$ is the commutative semigroup generated by all these elements subject to all relations except the last one. The result follows.

Lemma 1.6. For any $a, b \in A,\left(0 \otimes^{c} a\right)\left(1 \otimes^{c} b\right) \neq 0 \otimes^{c} a$ in $N_{0} \otimes^{c} A$.

Proof. In view of 1.5 we may prove that $(b, p(a))$, and $p(a)$ are never equivalent modulo $\mathscr{C}$. We shall prove a little more: that, if the class of $m \in M$ modulo $\mathscr{C}$ has more than one element, then $m \notin B$.

The proof uses the construction of the smallest congruence $\mathscr{C}$ on a semigroup $M$ which contains a given binary relation $\mathscr{R}$ on $M$. This construction will be used again later and is as follows. Let $\mathscr{B}$ be the binary relation on $M$ defined by $m \mathscr{B} n$ if and only if there exist $u, v \in M^{1}$ and $p, q \in M$ such that $m=u p v, n=u q v$ and either $p=q$ or $p \mathscr{R} q$ or $q \mathscr{R} p$. Then $\mathscr{C}$ is the transitive closure of $\mathscr{C}$ (cf. [2]).

In the particular situation of the lemma, let $m \in B$ be such that the class of $m$ modulo $\mathscr{C}$ has more than one element. Then there exists $n \in M$ such that $m \mathscr{B} n$, $m \neq n$. Write $m=u p, n=u q$, where $u \in M^{1}$ and either $p \mathscr{R} q$ or $q \mathscr{R} p$. Looking at the multiplication table of $M$ we observe that $p$ must be in $B$, which makes $p \mathscr{R} q$ and $q \mathscr{R} p$ impossible. Therefore, if $m \in B$, the class of $m$ modulo $\mathscr{C}$ cannot have more than one element, which completes the proof.

2. Functorial properties of the commutative tensor product. If $\left(A_{i}\right)_{i \in I},\left(B_{i}\right)_{i \in I}$ are families of commutative semigroups over the same index set $I$ and if, for each $i \in I$, $f_{i}$ is a homomorphism of $A$ into $B$, then there exists a unique homomorphism $\bigotimes_{i \in I}^{i} f_{i}$ of $\bigotimes_{i \in I}^{c} A_{i}$ into $\bigotimes_{i \in I}^{c} B_{i}$ such that $\left(\bigotimes_{i \in I}^{i} f_{i}\right)\left(\bigotimes_{i \in I}^{i} a_{i}\right)=\bigotimes_{i \in I}^{i} f_{i}\left(a_{i}\right)$ identically. This makes a multifunctor of $I$ variables, covariant in each variable, provided that we can select a commutative tensor product for each family (which is ensured by Theorem 2.1 of [5] and Theorem 1.1). Observe then that $\bigotimes_{i \in I} f_{i}=C\left(\bigotimes_{i \in I} f_{i}\right)$.

As was the case with $\otimes, A \otimes^{c} B$ and $B \otimes^{c} A$ are naturally equivalent bifunctors. The following property, however, does not hold for $\otimes$ [5, Example 2.3]:

Proposition 2.1. $A \otimes^{c} B \otimes^{c} C, A \otimes^{c}\left(B \otimes^{c} C\right)$ and $\left(A \otimes^{c} B\right) \otimes^{c} C$ are naturally equivalent trifunctors.

Proof. It parallels closely the proof of the similar property for, say, abelian groups. Therefore we prove only the natural equivalence of $A \otimes^{c} B \otimes^{c} C$ and $\left(A \otimes^{c} B\right) \otimes^{c} C$ in case the reader wants to see where the commutativity is used. For the same reason we leave out the more general forms of associativity of $\otimes^{c}$. 
Since the mapping $(a, b, c) \leadsto\left(a \otimes^{c} b\right) \otimes^{c} c$ is trilinear, there exists a homomorphism $u$ of $A \otimes^{c} B \otimes^{c} C$ into $\left(A \otimes^{c} B\right) \otimes^{c} C$ such that $u\left(a \otimes^{c} b \otimes^{c} c\right)=$ $\left(a \otimes^{c} b\right) \otimes^{c} c$ identically. On the other hand, $(a, b) \leadsto a \otimes^{c} b \otimes^{c} c$ is bilinear for each $c \in C$, whence there is a homomorphism $v_{c}$ of $A \otimes^{c} B$ into $A \otimes^{c} B \otimes^{c} C$ such that $v_{c}\left(a \otimes^{c} b\right)=a \otimes^{c} b \otimes^{c} c$ identically. If now $c^{\prime}, c^{\prime \prime} \in C$, then $v_{c^{\prime}} \cdot v_{c^{\prime \prime}}$ is also a homomorphism (since $A \otimes^{c} B \otimes^{c} C$ is commutative); it agrees with $v_{c^{\prime} c^{\prime \prime}}$ on all generators $a \otimes^{c} b$ of $A \otimes^{c} B$, which makes $v_{c}(t)$ a bilinear mapping of $\left(A \otimes^{c} B\right) \times C$ into $A \otimes^{c} B \otimes^{c} C$. Therefore there exists a homomorphism $v$ of $\left(A \otimes^{c} B\right) \otimes^{c} C$ into $A \otimes^{c} B \otimes^{c} C$ such that

$$
v\left(\left(a \otimes^{c} b\right) \otimes^{c} c\right)=v_{c}\left(a \otimes^{c} b\right)=a \otimes^{c} b \otimes^{c} c
$$

identically. It is immediate that $u$ and $v$ are inverse isomorphisms and are natural in $A, B, C$.

In order to obtain deeper properties of $\otimes^{c}$ we need a preliminary study of the functor $C$. First we say that a semigroup homomorphism $f$ of $A$ into $B$ is consistent if $b^{\prime} b^{\prime \prime} \in \operatorname{im} f$ implies $b^{\prime} \in \operatorname{im} f$ and $b^{\prime \prime} \in \operatorname{im} f$ (where $\operatorname{im} f$ is the range of $f$ ); i.e. if $B-\operatorname{im} f$ is either empty or an ideal (cf. [4], [2]).

LEMMA 2.2. If $f$ is consistent, then $C(f)$ is consistent; if furthermore $f$ is one-to-one, then $C(f)$ is one-to-one.

Proof. Let $f$ be of $A$ into $B$ and $p: A \rightarrow C(A), q: B \rightarrow C(B)$ be the projections, so that $C(f)$ is given by: $C(f) \circ p=q \circ f$. The congruence $\mathscr{C}=\operatorname{ker} p$ induced by $p$ is the smallest congruence on $A$ such that $a^{\prime} a^{\prime \prime} \mathscr{C} a^{\prime \prime} a^{\prime}$ identically; thus it is the transitive closure of the binary relation $\mathscr{C}_{1}$ on $A$ defined by: $a_{1} \mathscr{C}_{1} a_{2}$ if and only if there exist $u, v \in A^{1}, s, t \in A$ such that $a_{1}=u s v, a_{2}=u t v$ and either $s=t$ or $s=a^{\prime} a^{\prime \prime}, t=a^{\prime \prime} a^{\prime}$ for some $a^{\prime}, a^{\prime \prime} \in A$. The congruence $\mathscr{D}=\operatorname{ker} q$ has a similar description from the corresponding binary relation $\mathscr{D}_{1}$ on $B$.

Since $f$ is consistent, $b_{1} \mathscr{D}_{1} b_{2}$ and $b_{1} \in \operatorname{im} f$ implies $b_{2} \in \operatorname{im} f$. Therefore $b_{1} \mathscr{D} b_{2}$ and $b_{1} \in \operatorname{im} f$ implies $b_{2} \in \operatorname{im} f$. It follows that $C(f)$ is consistent: if $q\left(b^{\prime}\right) q\left(b^{\prime \prime}\right)$ $=C(f)(p(a))$ for some $a \in A$, then $b^{\prime} b^{\prime \prime} \mathscr{D} f(a)$, whence $b^{\prime}, b^{\prime \prime} \in \operatorname{im} f$ and $q\left(b^{\prime}\right), q\left(b^{\prime \prime}\right)$ $\in \operatorname{im} C(f)$.

If $f$ is also one-to-one, suppose that $C(f)\left(p\left(a^{\prime}\right)\right)=C(f)\left(p\left(a^{\prime \prime}\right)\right)$, where $a^{\prime}, a^{\prime \prime} \in A$. Then $f\left(a^{\prime}\right) \mathscr{D} f\left(a^{\prime \prime}\right)$ so that there exist $b_{1}, \ldots, b_{n} \in B$ such that $f\left(a^{\prime}\right)=b_{1}, b_{i} \mathscr{D}_{1} b_{i+1}$ for all $i=1, \ldots, n-1$ and $b_{n}=f\left(a^{\prime \prime}\right)$. By induction on $i$, there exist $a_{1}, \ldots, a_{n} \in A$ such that $b_{i}=f\left(a_{i}\right)$ for all $i$, by the first part of the proof. Now the assumptions on $f$ make that $a_{i} \mathscr{C}_{1} a_{i+1}$ for all $i=1, \ldots, n-1$. Therefore $a^{\prime} \mathscr{C} a^{\prime \prime}$, i.e. $p\left(a^{\prime}\right)=p\left(a^{\prime \prime}\right)$, which shows that $C(f)$ is one-to-one and completes the proof.

If $f$ and $g$ are one-to-one and consistent, then $f \otimes g$ is one-to-one and consistent [5, Theorem 4.1]. Then so is $f \otimes^{c} g=C(f \otimes g)$ by the lemma. This proves

THEOREM 2.3. If $f$ and $g$ are homomorphisms of commutative semigroups, and iff and $g$ are one-to-one and consistent, then $f \otimes^{c} g$ is one-to-one and consistent.

If on the other hand $f$ and $g$ are onto, then $f \otimes g$ is onto and so is $C(f \otimes g)=f \otimes^{c} g$. 
We defined in [5] a coexact sequence as a sequence $A \stackrel{f}{\rightarrow} B \stackrel{g}{\rightarrow} C$ of semigroups and homomorphisms such that $\operatorname{ker} g$ is the smallest congruence on $B$ some class of which contains $\operatorname{im} f$.

THEOREM 2.4. Let $A \stackrel{f}{\rightarrow} B \stackrel{g}{\rightarrow} C$ be a coexact sequence of commutative semigroups, where $g$ is consistent (for instance, onto). Let $X$ be an archimedean commutative semigroup. Then the sequence

$$
A \otimes^{c} X \stackrel{f \otimes^{c} \mathrm{id}_{x}}{\longrightarrow} B \otimes^{c} X \stackrel{g \otimes^{c} \mathrm{id}_{x}}{\longrightarrow} C \otimes^{c} X
$$

(where $\mathrm{id}_{X}$ is the identity on $X$ ) is coexact, and $g \otimes^{c} \mathrm{id}_{X}$ is consistent (onto, if $g$ is onto).

Proof. Since $E(X)$ is trivial, the sequence

$$
A \otimes X \stackrel{f \otimes \mathrm{id}_{X}}{\longrightarrow} B \otimes X \stackrel{g \otimes \mathrm{id}_{x}}{\longrightarrow} C \otimes X
$$

is coexact, with $g \otimes \mathrm{id}_{X}$ consistent, by Theorem 5.1 of [5].

Suppose first that $g$ is onto (so that $g \otimes \mathrm{id}_{X}$ is also onto). In this case the sequence of Theorem 2.4 is coexact with $g \otimes^{c} \mathrm{id}_{x}$ onto because $C$ preserves colimits. Indeed observe that a sequence $P \stackrel{u}{\rightarrow} Q \stackrel{v}{\rightarrow} R$ is coexact, with $v$ onto, if and only if $R$ is (with $v$ ) the colimit of the following diagram with three vertices (full arrows):

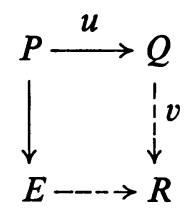

where $E$ is a one-element semigroup.

In the general case, $g$ can be written as $g=g^{\prime} \circ g^{\prime \prime}$, where $g^{\prime \prime}$ is onto and $g^{\prime}$ one-toone and consistent. Then the sequence $\left(f \otimes^{c} \mathrm{id}_{x}, g^{\prime \prime} \otimes^{c} \mathrm{id}_{x}\right)$ is coexact, with $g^{\prime \prime} \otimes^{c} \mathrm{id}_{X}$ onto, by the previous case. Also Theorem 2.3 implies that $g^{\prime} \otimes^{c} \mathrm{id}_{X}$ is consistent, so that $g \otimes^{c} \mathrm{id}_{x}=\left(g^{\prime} \otimes^{c} \mathrm{id}_{x}\right) \circ\left(g^{\prime \prime} \otimes^{c} \mathrm{id}_{X}\right)$ is consistent, and one-to-one, so that the sequence $\left(f \otimes^{c} \mathrm{id}_{X}, g \otimes^{c} \mathrm{id}_{X}\right)$ is coexact. This completes the proof.

As in the case of $\otimes$, it is possible to remove the assumption on $X$ by restricting the tensor product further, to the subcategory of commutative semigroups with zero (or identity) (see [5]).

Finally $\otimes$ has the following adjoint associativity property, that $\operatorname{Hom}(A \otimes B, C)$ and $\operatorname{Hom}(A, \operatorname{Hom}(B, C))$ are naturally isomorphic semigroups whenever $C$ is commutative (follows from [5, Theorem 5.6]).

TheOREM 2.5. For any commutative semigroups $A, B, C$, Hom $\left(A \otimes^{c} B, C\right)$ $\cong \operatorname{Hom}(A, \operatorname{Hom}(B, C))$ by an isomorphism which is natural in $A, B, C$.

Proof. We have the sequence of natural isomorphisms:

$$
\begin{aligned}
\operatorname{Hom}\left(A \otimes^{c} B, C\right) & \cong \operatorname{Hom}(C(A \otimes B), C) \cong \operatorname{Hom}(A \otimes B, C) \\
& \cong \operatorname{Hom}(A, \operatorname{Hom}(B, C)) .
\end{aligned}
$$


The two following lemmas will be of use. First let $\mathscr{C}(\mathscr{D})$ be a congruence on the commutative semigroup $A(B)$. Let $\mathscr{C} \otimes^{c} \mathscr{D}$ be the smallest congruence on $A \otimes^{c} B$ which contains all pairs $\left(a^{\prime} \otimes^{c} b, a^{\prime \prime} \otimes^{c} b\right)$ such that $\left(a^{\prime}, a^{\prime \prime}\right) \in \mathscr{C}$ and all pairs $\left(a \otimes^{c} b^{\prime}, a \otimes^{c} b^{\prime \prime}\right)$ such that $\left(b^{\prime \prime}, b^{\prime \prime}\right) \in \mathscr{D}$. A congruence $\mathscr{C} \otimes \mathscr{D}$ was defined analogously in [5, Definition 3.3], (using $\otimes$ instead of $\otimes^{c}$ ).

To deal with these congruences, it is useful to define, for each congruence $\mathscr{A}$ on a semigroup $S$, a congruence $C(\mathscr{A})$ on $C(S)$ as the smallest congruence on $C(S)$ which contains all pairs $\left(p\left(s^{\prime}\right), p\left(s^{\prime \prime}\right)\right)$ such that $\left(s^{\prime}, s^{\prime \prime}\right) \in \mathscr{A}$ (where $p$ is the projection of $S$ onto $C(S))$. Indeed it is clear that $C(\mathscr{C} \otimes \mathscr{D})=\mathscr{C} \otimes^{c} \mathscr{D}$. What we shall use is the following sublemma: if $f$ is a homomorphism of $S$ onto some semigroup $T$, then $C(\operatorname{ker} f)=\operatorname{ker} C(f)$.

Let $q$ be the projection of $T$ onto $C(T)$, so that $C(f) \circ p=q \circ f$. If $f\left(s^{\prime}\right)=f\left(s^{\prime \prime}\right)$, then $C(f)\left(p\left(s^{\prime}\right)\right)=C(f)\left(p\left(s^{\prime \prime}\right)\right)$, whence $C(\operatorname{ker} f) \subseteq \operatorname{ker} C(f)$. To obtain the converse inclusion, let $C=C(S) / C(\operatorname{ker} f)$ and $k$ be the projection of $C(S)$ onto $C$. Then $f\left(s^{\prime}\right)=f\left(s^{\prime \prime}\right)$ implies $k\left(p\left(s^{\prime}\right)\right)=k\left(p\left(s^{\prime \prime}\right)\right)$ by definition of $C(\operatorname{ker} f)$; hence $\operatorname{ker} f \subseteq$ ker $k \circ p$, so that $k \circ p=g \circ f$ for some homomorphism $g$ of $T$ into $C$, for $f$ is onto. Since $C$ is commutative, $g=u \circ q$ for some homomorphism $u$. Then

$$
k \circ p=g \circ f=u \circ q \circ f=u \circ C(f) \circ p
$$

so that $k=u \circ C(f)$ and $\operatorname{ker} C(f) \subseteq \operatorname{ker} k=C(\operatorname{ker} f)$. This proves our sublemma.

LEMMA 2.6. Let $f$ and $g$ be onto homomorphisms of commutative semigroups. Then $\operatorname{ker}\left(f \otimes^{c} g\right)=\operatorname{ker} f \otimes^{c} \operatorname{ker} g$.

Proof. By Theorem 3.4 of [5], $\operatorname{ker}(f \otimes g)=\operatorname{ker} f \otimes \operatorname{ker} g$, since $f$ and $g$ are onto. Since $f \otimes g$ is also onto, the sublemma yields

$\operatorname{ker}\left(f \otimes^{c} g\right)=\operatorname{ker} C(f \otimes g)=C(\operatorname{ker} f \otimes g)=C(\operatorname{ker} f \otimes \operatorname{ker} g)=\operatorname{ker} f \otimes^{c} \operatorname{ker} g$.

LEMMA 2.7. Let $\mathscr{P}(\mathscr{Q})$ be a binary relation on the commutative semigroup $A(B)$ and $\mathscr{C}(\mathscr{D})$ be the smallest congruence on $A(B)$ containing $\mathscr{P}(\mathscr{Q})$. Then $\mathscr{C} \otimes^{c} \mathscr{D}$ is the smallest congruence on $A \otimes^{c} B$ which contains all pairs $\left(a^{\prime} \otimes^{c} b, a^{\prime \prime} \otimes^{c} b\right)$ such that $\left(a^{\prime}, a^{\prime \prime}\right) \in \mathscr{P}$ and all pairs $\left(a \otimes^{c} b^{\prime}, a \otimes^{c} b^{\prime \prime}\right)$ such that $\left(b^{\prime}, b^{\prime \prime}\right) \in \mathscr{Q}$.

Proof. Same as the proof of Lemma 3.6 of [5].

We are now in position to prove that $\otimes^{c}$ preserves colimits. First, we have

THEOREM 2.8. Let $X$ be any commutative semigroup. The functor $A m A \otimes^{c} X$ preserves direct sums. More precisely, if $\left(A_{i}\right)_{i \in I}$ is any family of commutative semigroups, there exists an isomorphism $\theta$ of $\left(\coprod_{i \in I} A_{i}\right) \otimes^{c} X$ onto $\coprod_{i \in I}\left(A_{i} \otimes^{c} X\right)$ which is natural in $X$ and $\left(A_{i}\right)_{i \in I}$ and such that $\theta \circ\left(m_{i} \otimes^{c} \mathrm{id}_{X}\right)=n_{i}$ for all $i \in I$, where $m_{i}\left(n_{i}\right)$ is the injection of $A_{i}\left(A_{i} \otimes^{c} X\right)$ into $\coprod_{i \in I} A_{i}\left(\coprod_{i \in I}\left(A_{i} \otimes^{c} X\right)\right)$.

Proof. For some descriptions of the direct sum, see [2] (the direct sum is there denoted by $\Sigma$ ). All we use is the fact that it is a direct sum in the category of all commutative semigroups. 
Observe that $\left(a_{i}, x\right) \leadsto m_{i}\left(a_{i}\right) \otimes^{c} x$ is a bilinear mapping of $A_{i} \times X$ into $\left(\coprod_{i \in I} A_{i}\right)$ $\otimes^{c} X$. Hence it induces for each $i$ a homomorphism $\varphi_{i}$ of $A_{i} \otimes^{c} X$ into ( $\coprod_{i \in I} A_{i}$ ) $\otimes^{c} X$, whence there is a homomorphism $\varphi$ of $\coprod_{i \in I}\left(A_{i} \otimes^{c} X\right)$ into $\left(\coprod_{i \in I} A_{i}\right) \otimes^{c} X$ such that $\varphi\left(n_{i}\left(a_{i} \otimes^{c} x\right)\right)=m_{i}\left(a_{i}\right) \otimes^{c} x$ identically.

On the other hand, for every $i \in I, x \in X, \theta_{i, x}\left(a_{i}\right)=n_{i}\left(a_{i} \otimes x\right)$ defines a homomorphism of $A_{i}$ into $\bigsqcup_{i \in I}\left(A_{i} \otimes^{c} X\right)$, whence there is, for each $x \in X$, a homomorphism $\theta_{x}$ of $\bigsqcup_{i \in I} A_{i}$ into $\bigsqcup_{i \in I}\left(A_{i} \otimes^{c} X\right)$ such that $\theta_{x}\left(m_{i}\left(a_{i}\right)\right)=n_{i}\left(a_{i} \otimes^{c} x\right)$. Since $\bigsqcup_{i \in I}\left(A_{i} \otimes^{c} X\right)$ is commutative, the pointwise product $\theta_{x^{\prime}} \cdot \theta_{x^{\prime \prime}}$ is a homomorphism for any $x^{\prime}, x^{\prime \prime} \in X$; it agrees with $\theta_{x^{\prime} x^{\prime \prime}}$ on each generator $m_{i}\left(a_{i}\right)$ of $\coprod_{i \in I} A_{i}$, whence $\theta_{x^{\prime} x^{\prime \prime}}=\theta_{x^{\prime}} \cdot \theta_{x^{\prime \prime}}$. This proves that $\theta_{x}(a)$ is a bilinear function of $(a, x)$. Therefore there exists a homomorphism $\theta$ of $\left(\amalg_{i \in I} A_{i}\right) \otimes^{c} X$ into $\amalg_{i \in I}\left(A_{i} \otimes^{c} X\right)$ such that $\theta\left(m_{i}\left(a_{i}\right) \otimes^{c} x\right)=n_{i}\left(a_{i} \otimes^{c} x\right)$ identically.

It is now clear that $\theta$ and $\varphi$ are inverse isomorphisms and that $\theta$ has all the properties required in the theorem.

Observe that a very short proof of this theorem can be given, using adjoint associativity (Theorem 2.5), but it will not give the formula $\theta \circ\left(m_{i} \otimes^{c} \mathrm{id}_{\bar{X}}\right)=n_{i}$ which we shall need later (and does not follow from the naturality in this case!).

THEOREM 2.9. Let $X$ be any commutative semigroup. The functor $A \leadsto A \otimes^{c} X$ preserves colimits.

Proof. Let $D$ be a diagram of commutative semigroups, i.e. a family $\left(A_{i}\right)_{i \in I}$ of commutative semigroups with, for each $i, j \in I$, a (perhaps empty) family $\left(f_{m}\right)_{m \in M_{i, j}}$ of homomorphisms of $A_{i}$ into $A_{j}$. The colimit of $D$ consists of a commutative semigroup $A$ and a family $\left(f_{i}\right)_{i \in I}$ of homomorphisms $f_{i}$ of $A_{i}$ into $A$, and it can be constructed as follows: let $n_{i}$ be the injection of $A_{i}$ into $\bigsqcup_{i \in I} A_{i}$ and $\mathscr{C}$ be the smallest congruence on $\coprod_{i \in I} A_{i}$ which contains all pairs $\left(n_{j}\left(f_{m}\left(a_{i}\right)\right), n_{i}\left(a_{i}\right)\right)$ such that $a_{i} \in A_{i}$ and $m \in M_{i, j}$; then we may take $A=\coprod_{i \in I} A_{i} / \mathscr{C}$ and $f_{i}=k \circ n_{i}$, where $k$ is the projection of $\coprod_{i \in I} A_{i}$ onto $A$.

To prove that $A \otimes^{c} X$ together with the family $\left(f_{i} \otimes^{c} I_{X}\right)_{i \in I}$ is a colimit of the diagram $D \otimes^{c} X$, we shall verify that our functor preserves each step of the construction above, up to isomorphism. Theorem 2.8 gives an isomorphism $\theta$ of $\left(\coprod_{i \in I} A_{i}\right) \otimes^{c} X$ onto $\coprod_{i \in I}\left(A_{i} \otimes^{c} X\right)$ such that $\theta \circ\left(n_{i} \otimes^{c} I_{X}\right)$ is the injection $q_{i}$ of $A_{i} \otimes^{c} X$ into $\amalg_{i \in I}\left(A_{i} \otimes^{c} X\right)$, which is a first step.

Next $k$ yields a homomorphism $\bar{R}=\left(k \otimes^{c} \mathrm{id}_{X}\right) \circ \theta^{-1}$ of $\coprod_{i \in I}\left(A_{i} \otimes^{c} X\right)$ onto $A \otimes^{c} X$; it follows from $2.6,2.7$ that $\operatorname{ker} \bar{k}$ is the smallest congruence on $\bigsqcup_{i \in I}\left(A_{i} \otimes^{c} X\right)$ which contains all pairs $\left(q_{j}\left(\left(f_{m} \otimes^{c} \mathrm{id}_{X}\right)\left(a_{i} \otimes^{c} x\right)\right), q_{i}\left(a_{i} \otimes^{c} x\right)\right)$ such that $a_{i} \in A_{i}, m \in M_{i, j}\left(\right.$ since $\left.\theta \circ\left(n_{i} \otimes^{c} \mathrm{id}_{X}\right)=q_{i}\right)$.

Finally $f_{i}$ yields a homomorphism $f_{i} \otimes^{c}$ id $_{X}$ of $A_{i} \otimes^{c} X$ into $A \otimes^{c} X$ such that

$$
f_{i} \otimes^{c} \mathrm{id}_{x}=\left(k \otimes^{c} \mathrm{id}_{x}\right) \circ\left(n_{i} \otimes^{c} \mathrm{id}_{x}\right)=\bar{k} \circ \theta \circ\left(n_{i} \otimes^{c} \mathrm{id}_{x}\right)=\bar{k} \circ q_{i} .
$$

This completes the proof of the theorem. 
3. Flat commutative semigroups. A commutative semigroup $K$ is flat if $f \otimes^{c} \mathrm{id}_{K}$ is one-to-one whenever $f$ is a one-to-one homomorphism of commutative semigroups. For instance,

Proposition 3.1. Any free commutative semigroup is flat.

Proof. Let $F$ be a free commutative semigroup on some set $I \neq \varnothing$; we can write $F$ as a direct sum $\coprod_{i \in I} N_{i}$ of infinite cyclic semigroups. Let also $A$ and $B$ be commutative semigroups and $f$ be a one-to-one homomorphism of $A$ into $B$. Theorem 2.8 provides an isomorphism $\theta_{A}$ of $A \otimes^{c} F$ onto $\bigsqcup_{i \in I} A_{i}$, where $A_{i}=A \otimes^{c} N_{i} \cong A$, and an isomorphism $\theta_{B}$ of $B \otimes^{c} F$ onto $\bigsqcup_{i \in I} B_{i}$, where $B_{i} \cong B$. The homomorphism $f$ induces a homomorphism $f_{i}$ of $A_{i}$ into $B_{i}$ which is one-to-one, for all $i \in I$; now $\coprod_{i \in I} f_{i}$ is also one-to-one and, since $\theta_{B} \circ\left(f \otimes^{c} \mathrm{id}_{F}\right)=\left(\coprod_{i \in I} f_{i}\right) \circ \theta_{A}$ by naturality, $f \otimes^{c} \mathrm{id}_{F}$ is one-to-one.

From other results of $\S 1$ we can obtain some properties of flat commutative semigroups.

Proposition 3.2. Any flat commutative semigroup $K$ is separative.

Proof. Separative is equivalent to: each archimedean component of $K$ is cancellative, and to: $K$ can be embedded into a union of groups (see, for instance [1]). We prove the latter. Let $N(Z)$ be the additive semigroup of all positive integers (all integers); the embedding of $N$ into $Z$ yields, if $K$ is flat, an embedding of $N \otimes^{c} K \cong K$ into $Z \otimes^{c} K$, which is a union of groups by 1.4 .

Proposition 3.3.' Let $K$ be a flat commutative semigroup; for any $x, y \in K$, $x \neq x y$. In particular a flat commutative semigroup contains no idempotent.

Proof. Let $N_{0}$ be the additive semigroup of all nonnegative integers and $Z$ be as above. If $K$ is flat, the embedding of $N_{0}$ into $Z$ yields an embedding of $N_{0} \otimes^{c} K$ into $Z \otimes^{c} K$. Let $x, y \in K$; by $1.6,0 \otimes^{c} x \neq\left(0 \otimes^{c} x\right)\left(1 \otimes^{c} y\right)$ in $N_{0} \otimes^{c} K$. Yet if $x y=x$, then we have in $Z \otimes^{c} K$ the equality

$\left(0 \otimes^{c} x\right)\left(1 \otimes^{c} y\right)=\left(-1 \otimes^{c} x\right)\left(1 \otimes^{c} x\right)\left(1 \otimes^{c} y\right)=\left(-1 \otimes^{c} x\right)\left(1 \otimes^{c} x\right)=0 \otimes^{c} x$, which is impossible if $K$ is flat.

It follows from 3.3 that a flat (=torsionless) abelian group is not flat in the category of commutative semigroups. In fact, flatness is a fairly strong condition for a commutative semigroup, as shown by the following

THEOREM 3.4. Let $K$ be a commutative semigroup having a minimal generating subset $X$. Then $K$ is flat if and only if it is free.

Proof. The converse is just 3.1. To prove the direct part, we assume that $K$ is flat, but not free. In this case, $K$ is not free on $X$, so that a nontrivial relation holds in $K$ between some elements of $X$; we can always suppose that this relation is of the form

$$
x_{1}^{p_{1}} x_{2}^{p_{2}} \cdots x_{n^{n}}^{p_{n}}=x_{1}^{q}{ }_{1} x_{2}^{q} \cdots x_{n}^{q},
$$


where the $p_{i}$ 's and $q_{i}$ 's are positive integers. Since the relation is not trivial there exist indices such that $p_{i} \neq q_{i}$ and we may further assume that these come first, that is that there is an integer $m, 1 \leqq m \leqq n$ such that $p_{i} \neq q_{i}$ whenever $i \leqq m$ and $p_{i}=q_{i}$ whenever $i>m$. Also we may assume that $n>1$; if $n=1$, then $K$ contains an idempotent, which is impossible by 3.3 .

Let $B$ be the free commutative semigroup on $n+1$ generators $b, b_{1}, b_{2}, \ldots, b_{n}$. Consider the elements $a_{i}=b_{i} b^{p_{i}}, a_{i}^{\prime}=b_{i} b^{a_{i}}$; these are $n+m$ elements of $B$ since $a_{i}=a_{i}^{\prime}$ whenever $i>m$. In $B \otimes^{c} K$ we have

$$
\begin{aligned}
\left(a_{1} \otimes^{c} x_{1}\right) \cdots\left(a_{n} \otimes^{c} x_{n}\right) & =\left(b_{1} b^{p_{1}} \otimes^{c} x_{1}\right) \cdots\left(b_{n} b^{p_{n}} \otimes^{c} x_{n}\right) \\
& =\left(b_{1} \otimes^{c} x_{1}\right) \cdots\left(b_{n} \otimes^{c} x_{n}\right)\left(b^{p_{1}} \otimes^{c} x_{1}\right) \cdots\left(b^{p_{n}} \otimes^{c} x_{n}\right) \\
& =\left(b_{1} \otimes^{c} x_{1}\right) \cdots\left(b_{n} \otimes^{c} x_{n}\right)\left(b \otimes^{c} x_{1}^{p_{1}} \cdots x_{n^{n}}^{p_{n}}\right) \\
& =\left(b_{1} \otimes^{c} x_{1}\right) \cdots\left(b_{n} \otimes^{c} x_{n}\right)\left(b \otimes^{c} x_{1}^{q_{1}} \cdots x_{n_{n}}^{q_{n}}\right) \\
& =\cdots=\left(b_{1} b^{q_{1}} \otimes^{c} x_{1}\right) \cdots\left(b_{n} b^{q_{n}} \otimes^{c} x_{n}\right) \\
& =\left(a_{1}^{\prime} \otimes^{c} x_{1}\right) \cdots\left(a_{n}^{\prime} \otimes^{c} x_{n}\right) .
\end{aligned}
$$

Now we proceed to show that $\left(a_{1} \otimes^{c} x_{1}\right) \cdots\left(a_{n} \otimes^{c} x_{n}\right)$ and $\left(a_{1}^{\prime} \otimes^{c} x_{1}\right)$ $\cdots\left(a_{n}^{\prime} \otimes^{c} x_{n}\right)$ are different in $A \otimes^{c} K$, where $A$ is the subsemigroup of $B$ generated by the $a_{i}$ 's and $a_{i}^{\prime \prime}$. This will contradict the assumption that $K$ is flat and complete the proof. This would be very easy if $A$ were free (which can be shown to happen only for $m=1$ ). As it may not be the case, we seek a presentation of $A$. The following presentation will serve, even though it could be replaced by a finite presentation, since $A$ is finitely generated [7].

Lemma 3.5. The semigroup $A$ is isomorphic to the commutative semigroup generated by $a_{1}, \ldots, a_{n}, a_{1}^{\prime}, \ldots, a_{m}^{\prime}$ subject to all relations which hold in $A$ and have the form

$$
a_{1}^{s_{1}} \cdots a_{m}^{s_{m}} a_{1}^{s_{1}^{\prime}} \cdots a_{m}^{s_{m}^{\prime}}=a_{1}^{s_{1}^{\prime}} \cdots a_{m}^{s_{s}^{\prime}} a_{1}^{s_{1}} \cdots a_{m}^{s_{m}}
$$

where, for each $i \leqq m$, either $s_{i}=0$ or $s_{i}^{\prime}=0 ; a_{1}^{0}, a_{i}^{\prime 0}$ are considered as a formal identity; for some $i \leqq m$, either $s_{i} \neq 0$ or $s_{i}^{\prime} \neq 0$.

Proof. It is enough to show that any relation between $a_{1}, \ldots, a_{n}, a_{1}^{\prime}, \ldots, a_{m}^{\prime}$ is a consequence of one of these. Suppose that

$$
a_{1}^{t_{1}} \cdots a_{n}^{t_{n}} a_{1}^{\prime t_{1}^{\prime}} \cdots a_{m}^{\prime t^{\prime}{ }^{\prime}}=a_{1}^{u_{1}} \cdots a_{n}^{u_{n}} a_{1}^{\prime u_{1}^{\prime}} \cdots a_{m}^{\prime u_{m}^{\prime}}
$$

holds in $A$, where $t_{i}, t_{i}^{\prime}, u_{i}, u_{i}^{\prime}$ are nonnegative integers but not all zero, and that $t_{i} \neq u_{i}$ or $t_{i}^{\prime} \neq u_{i}^{\prime}$ for some $i$, so that the relation is not trivial. Since $B$ is free on $b, b_{1}, \ldots, b_{n}$, one must have $t_{i}+t_{i}^{\prime}=u_{i}+u_{i}^{\prime}$ for all $i \leqq m, t_{i}=u_{i}$ for all $i>m$. Since $A$ is cancellative, we obtain by cancellation a relation of the form described in the lemma, which holds in $A$, and of which the given relation is a consequence since it can be obtained by multiplying both sides by some element of $A$.

Let $\mathscr{R}$ be the set of all relations described in 3.5. This presentation of $A$ gives a homomorphism $\varphi$ of the free commutative semigroup $F$ generated by $a_{1}, \ldots, a_{n}, a_{1}^{\prime}$, 
$\cdots, a_{m}^{\prime}$ onto $A$, such that $\operatorname{ker} \varphi$ is the smallest congruence on $F$ which contains $\mathscr{R}$. Since $F$ is the direct sum of all (infinite cyclic) subsemigroups $N_{a_{i}}, N_{a_{i}}$ generated in $F$ by $a_{i}, a_{i}$, Theorem 2.8 gives an isomorphism $\theta$ of $F \otimes^{c} K$ onto $K_{a_{1}} \amalg K_{a_{2}} \amalg$ $\cdots \amalg K_{a_{n}} \amalg K_{a_{1}^{\prime}} \amalg \cdots \amalg K_{a_{m}^{\prime}}$, where $K_{a_{i}}=N_{a_{i}} \otimes^{c} K$ is isomorphic to $K$ by $y m \rightarrow a_{i}$ $\otimes^{c} y=y_{a_{i}}$, and similarly for $K_{a_{i}}$. Then we have a homomorphism $\psi=\left(\varphi \otimes^{c} \mathrm{id}_{K}\right)$ ○ $\theta^{-1}$ of $K_{a_{1}} \amalg \cdots \amalg K_{a_{n}} \amalg K_{a_{i}^{\prime}} \amalg \cdots \amalg K_{a_{m}^{\prime}}=S$ onto $A \otimes^{c} K$; by $2.6,2.7, \operatorname{ker} \psi=\mathscr{C}$ is the smallest congruence on the direct sum which contains all pairs

such that

$$
\left(y_{a_{1}}^{s_{1}} \cdots y_{a_{m}}^{s_{m}} y_{a_{1}^{\prime}}^{s_{1}^{\prime}} \cdots y_{a_{m}^{\prime}}^{s_{m}^{\prime}}, y_{a_{1}}^{s_{1}^{\prime}} \cdots y_{a_{m}}^{s_{m}^{\prime}} y_{a_{1}^{\prime}}^{s_{1}} \cdots y_{a_{m}^{\prime}}^{s_{m}}\right)
$$

$$
a_{1}^{s_{1}} \cdots a_{m}^{s_{m}} a_{1}^{s_{1}^{\prime}} \cdots a_{m}^{s_{m}^{\prime}}=a_{1}^{s_{1}^{\prime}} \cdots a_{m}^{s_{m}^{\prime}} a_{1}^{s_{1}} \cdots a_{m}^{s_{m}}
$$

is one of the relations described in 3.5, and $y \in K$.

To show that $\left(a_{1} \otimes^{c} x_{1}\right) \cdots\left(a_{n} \otimes^{c} x_{n}\right)$ and $\left(a_{1}^{\prime} \otimes^{c} x_{1}\right) \cdots\left(a_{n}^{\prime} \otimes^{c} x_{n}\right)$ are different in $A \otimes^{c} K$ is now equivalent to proving that $\left(x_{1}\right)_{a_{1}} \cdots\left(x_{n}\right)_{a_{n}}$ and $\left(x_{1}\right)_{a_{1}^{\prime}} \cdots\left(x_{n}\right)_{a_{n}^{\prime}}$ are not equivalent modulo $\mathscr{C}$ in $S$. This is done as follows. If they are equivalent, then there will exist $v_{1}, \ldots, v_{r} \in S$ such that $v_{1}=\left(x_{1}\right)_{a_{1}} \cdots\left(x_{n}\right)_{a_{n}}, v_{r}=\left(x_{1}\right)_{a_{1}^{1}} \cdots\left(x_{n}\right)_{a_{n}^{\prime}}$ and that $v_{j}, v_{j+1}$ are related as follows: either $v_{j}=v_{j+1}$ or there exist $w_{j} \in S^{1}$ and a relation of the kind described in 3.5:

$$
a_{1}^{s_{1}} \cdots a_{m}^{s_{m}} a_{1}^{s_{1}^{\prime}} \cdots a_{m}^{s_{m}^{\prime}}=a_{1}^{s_{1}^{\prime}} \cdots a_{m}^{s_{m}^{\prime}} a_{1}^{s_{1}} \cdots a_{m}^{s_{m}}
$$

such that

$$
\begin{aligned}
v_{j} & =w_{j} y_{a_{1}}^{s_{1}} \cdots y_{a_{m}}^{s_{m}} y_{a_{1}^{\prime}}^{s_{1}^{\prime}} \cdots y_{a_{m}^{\prime}}^{s_{m}^{\prime}}, \\
v_{j+1} & =w_{j} y_{a_{1}}^{s_{1}^{\prime}} \cdots y_{a_{m}}^{s_{m}^{\prime}} y_{a_{1}^{\prime}}^{s_{1}} \cdots y_{a_{m}^{\prime}}^{s_{m}}
\end{aligned}
$$

for some $y \in K$ (observe that the roles of the $s_{i}$ 's and $s_{i}^{\prime}$ 's can be exchanged).

Suppose first that $v_{1} \neq v_{2}$. By the condition on $v_{1}$, we must have $s_{1}^{\prime}=\cdots=s_{m}^{\prime}=0$ in the direct sum $S$; furthermore, equating the components in $K_{a_{i}}$ yields $x_{i}=z_{i} y^{s_{i}}$ for all $i \leqq m$, where $z_{i} \in K^{1}$ is the component of $w_{1}$. Since $K$ is generated by $X$, we obtain for each $i \leqq m$ a relation between the elements of $X$.

Suppose that this relation is trivial for all $i \leqq m$. Then for each $i \leqq m, z_{i}=1, y=x_{i}$ and $s_{i}=1$, or else $z_{i}=x_{i}, s_{i}=0$. If there is only one $i \leqq m$ such that $s_{i} \neq 0$, (1) reads: $a_{i}=a_{i}^{\prime}$, which is impossible since $p_{i} \neq q_{i}$ as $i \leqq m$. If there exist $i, j \leqq m, i \neq j$ such that $s_{i} \neq 0, s_{j} \neq 0$, then $x_{i}=y=x_{j}$, which is impossible. Therefore, we can find $i \leqq m$ such that $x_{i}=z_{i} y^{s_{i}}$ is a nontrivial relation between the elements of $X$.

Choosing $i$ thus, this relation expresses $x_{i}$ as a product of elements of $X$. It is impossible that this product does not contain $x_{i}$, since $X$ is a minimal generating subset. But if it contains $x_{i}$, then we have in $K$ an equality of the form $x_{i}=x_{i} t$ for some $t \in K$, which is impossible by 3.3.

Thus $v_{1}=v_{2}$. By induction on $j$, we obtain similarly that $v_{1}=v_{j}$ for all $j \leqq r$, in particular $v_{1}=v_{r}$. But the conditions on $v_{1}, v_{r}$ make such equality impossible in the direct sum $S$.

This completes the proof of the theorem. 
There are a few more things we can say about flatness. First we have the following consequences of Theorem 3.4.

Proposition 3.6. For commutative semigroups, flatness is not hereditary.

Proof. Let $F$ be the free commutative semigroup on two generators $a, b$. Then $F$ is flat by 3.1. However, the ideal $A$ of $F$ consisting of all elements of the form $a^{n} b^{m}$, where $n, m>0$, is not flat; for it has a minimal generating subset

$$
X=\left\{a b^{m}, a^{n} b ; m, n>0\right\},
$$

yet it is not free since it is not free on $X$.

COROLlaRY 3.7. There exists a commutative semigroup $X$ such that $f \otimes^{c} \mathrm{id}_{X}$ is one-to-one whenever $f$ is a one-to-one homomorphism of a free commutative semigroup $A$ into a commutative semigroup $B$, yet which is not flat.

Proof. Suppose it were not so; then any commutative semigroup having the property indicated in the statement would be flat. Then any subsemigroup $L$ of any flat semigroup $K$ would be flat, since, for any one-to-one homomorphism $f$ of a free commutative semigroup $A$ into a commutative semigroup $B$, the commutativity of the diagram

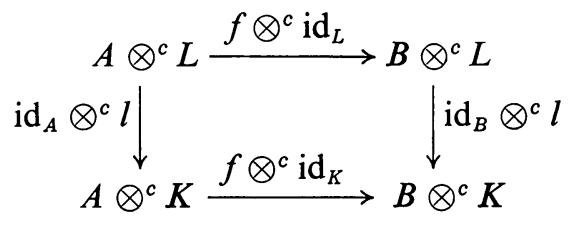

(where $l$ is the inclusion of $L$ in $K$ ) and the flatness of $K$ and $A$ imply that $f \otimes^{c} \operatorname{id}_{L}$ is one-to-one. But this contradicts 3.6.

In particular, the proof of Theorem 3.4 cannot be shortened by a more ingenious choice of $A$ and $B$ in which $A$ would be free.

There is, however, a weak form of hereditary property which applies to flatness.

Proposition 3.8. Any consistent subsemigroup of a flat commutative semigroup is flat.

Proof. Let $K$ be flat, and consider the diagram in the proof of 3.7, where now we do not suppose that $A$ is free, but take $L$ consistent. Then $\operatorname{id}_{A} \otimes^{c} l$ is one-to-one by Theorem 2.3 and the commutativity of the diagram yields that $L$ is flat.

Finally, 3.1 will yield more examples of flat commutative semigroups, due to the following result:

Proposition 3.9. For commutative semigroups, flatness is a local property.

Proof. This means that any locally flat commutative semigroup is flat (see [3]). Let $K$ be locally flat, so that $K$ is the union of a directed family $\left(K_{i}\right)_{i \in I}$ of flat (commutative) subsemigroups of $K$. The inclusion relations between the $K_{i}$ preorder $I$ in the obvious manner, and then $I$ becomes a directed preordered set and the 
family $\left(K_{i}\right)_{i \in I}$ together with the inclusion homomorphisms becomes a directed $I$-system; then $K$ is the inductive (or direct) limit of the system: $K=\operatorname{inj} \lim K_{i}$.

Let $A$ and $B$ be commutative semigroups and $f$ be a one-to-one homomorphism of $A$ into $B$. Since each $K_{i}$ is flat, $f \otimes^{c}$ id $_{K_{i}}$ is one-to-one for all $i \in I$; but the $A \otimes^{c} K_{i}$, $B \otimes{ }^{c} K_{i}$ form directed $I$-systems, and, taking the inductive limit, we obtain a oneto-one homomorphism inj $\lim \left(f \otimes^{c} \mathrm{id}_{K_{i}}\right)$ of inj $\lim \left(A \otimes^{c} K_{i}\right)$ into inj $\lim \left(B \otimes^{c} K_{i}\right)$. Finally the tensor product preserves colimits (Theorem 2.9), in particular inductive limits, which yields natural isomorphisms inj $\lim \left(A \otimes^{c} K_{i}\right) \cong A \otimes^{c} K$,

$$
\text { inj } \lim \left(B \otimes^{c} K_{i}\right) \cong B \otimes^{c} K
$$

and a commutative diagram

$$
\begin{gathered}
\operatorname{inj} \lim \left(A \otimes^{c} K_{i}\right) \stackrel{\operatorname{inj} \lim \left(f \otimes^{c} \mathrm{id}_{K_{i}}\right)}{\longrightarrow} \operatorname{inj} \lim \left(B \otimes^{c} K_{i}\right) \\
A \otimes^{c} K \stackrel{f \otimes^{c} \operatorname{id}_{K} \longrightarrow 2}{\longrightarrow} B \otimes^{c} K
\end{gathered}
$$

from which follows that $f \otimes^{c} \mathrm{id}_{K}$ is one-to-one, which completes the proof.

COROLlaRY 3.10. Any locally free commutative semigroup is flat.

Consider for instance the additive semigroup $Q$ of all positive rational numbers. This semigroup is locally free, in fact locally infinite cyclic; the subsemigroups of the local system can be taken as the subsemigroups $Q_{n}=\{p / n ; p>0\}$; these form a directed family since $Q_{n} \cup Q_{m} \subseteq Q_{n m}$. Therefore $Q$ is flat. Observe that it is not free.

Problem. Is it true that a commutative semigroup is flat if and only if it is locally free?

Added in proof. We also wish to acknowledge that the existence of the tensor product of commutative semigroups, and some of its properties (in particular 1.3, 1.4) were discovered independently by T. J. Head (J. Natur. Sci. and Math. 7 (1967), 39-49).

\section{REFERENCES}

1. A. H. Clifford and G. B. Preston, The algebraic theory of semigroups, Vol. 1, Math. Surveys, No. 7, Amer. Math. Soc., Providence, R. I., 1961; reprint 1964.

2. - — The algebraic theory of semigroups, Vol. 2, Math. Surveys, No. 7, Amer. Math. Soc., Providence, R. I., 1967.

3. P. M. Cohn, Universal algebra, Harper and Row, New York, 1965.

4. P. Dubreil, Contribution à la théorie des demi-groupes, Mém. Acad. Sci. Inst. France (2) 63 (1941), $52 \mathrm{pp}$.

5. P. A. Grillet, The tensor product of semigroups, Trans. Amer. Math. Soc. 138 (1969), 267-280.

6. B. Mitchell, Theory of categories, Academic Press, New York, 1965.

7. L. Rédei, Theorie der endlich erzeugbaren kommutativen Halbgruppen, Hamburger Math. Einzelschriften, No. 41, Physica-Verlag, Würzburg, 1963.

8. N. Kimura, Identity $(x y)^{n}=x^{n} y^{n}$ on semigroups, (to appear).

Kansas State University, ManhatTan, Kansas 